\title{
High-speed pulse train generation by spectral filtering of a mode-locked laser output
}

\author{
Zhang Jia, Hong Song-Jin and Seo Dong-Sun \\ Department of Electronic Engineering, Myongji University, San 38-2, Namdong, \\ Churingu, Yongin, Gyunggido 449-728, Korea, e-mail: sdsphoto@mju.ac.kr
}

Received: 13.01 .2010

\begin{abstract}
We demonstrate a simple method for generating high-speed pulse trains from a relatively low-speed pulse source by spectral filtering using a high-finesse fibre Fabry-Pérot interferometer. Reasonably stable $40 \mathrm{GHz}$ and $100 \mathrm{GHz}$ pulse trains are obtained at $1550 \mathrm{~nm}$ from a $10 \mathrm{GHz}$ mode-locked fibre ring laser.

PACS: $42.65 . \mathrm{Re}+42.25 . \mathrm{Hz}$

UDC: 535.41

Keywords: optical pulses, repetition rate multiplication, spectral filtering, ultrafast technology
\end{abstract}

\section{Introduction}

High-speed optical pulses are very attractive for many applications, such as microwave/millimeter-wave photonics, high-speed optical-time-division-multiplexing optical communications, high-speed optical sampling, etc. Active mode locking of fibre ring lasers is an efficient technique for generating stable picosecond pulses with low-timing jitter at several GHz. However, the maximum pulse speed of actively mode-locked fibre lasers (MLFLs) is practically limited by the bandwidth of modulators and/or their driving electronics. It is therefore a challenging problem to find ways for increasing repetition speed of the output pulses from an actively MLFL, without increasing the intensity or phase modulation frequency.

Several techniques for increasing the pulse speed by repetition rate multiplication have already been suggested. A rational harmonic mode-locking technique has offered successful generation of optical pulses at several times of the driving frequency of intensity modulator $[1,2]$. However, the output has shown serious periodic pulse amplitude fluctuations with increasing multiplication factor. Intra-cavity filtering or higher-order frequency modulation mode-locking based on high-finesse Fabry-Pérot interferometers has resulted in a good stability $[3,4]$. However, the multiplication factor has been relatively small and the inserted interferometer has induced large laser cavity loss.

Without changing anything inside the laser cavity, high-speed pulse trains could also be generated by simply passing a mode-locked laser output through a device or apparatus for repetition rate multiplication, such as a dispersive fibre [5], fibre Bragg grating [6], a time-to-space pulse shaper [7], etc. Those methods also suffer from some drawbacks, such 
as pulse-to-pulse spectrum change $[4,5]$ and/or relatively large amplitude variation [6-8]. An arrayed waveguide grating with a relatively narrow free spectral range (FSR) has been used as an extra-cavity repetition rate multiplier by periodic spectral filtering of ultrashort pulses in its FSR [8]. Physically small FSR implemented by large (larger than the input pulse width) time-delay increment per waveguide split individual input pulses into multiple pulses to achieve repetition rate multiplication corresponding to the number of waveguides. Even though successful repletion rate multiplication has been achieved in the work [8], each pass-band spectrum has shown side bands, due to a wide bandwidth of the arrayed waveguide grating filter, resulting in pulse burst (rather than continuous pulse train) with the period of input pulses.

Repetition rate multiplication by double-passing a low-finesse Fabry-Pérot interferometer has also been reported [9]. The amplitude fluctuation in the output pulse train has been reduced at the cost of extra components (Faraday rotator, polarising beam splitter, polarisation controller, etc.) for doubly passing the Fabry-Pérot interferometer. In the present study we use a high-finesse fibre Fabry-Pérot interferometer (FFPI) for a single pass but similar sharp spectral filtering, ensuring stable pulse train generation within its FSR. As a result, we obtain reasonably stable $40 \mathrm{GHz}$ and $100 \mathrm{GHz}$ pulse trains from a $10 \mathrm{GHz}$ mode-locked fibre.

\section{Experimental setup}

Fig. 1 shows a conceptual diagram of high-speed pulse train generation from a relatively slow pulse source by extra-cavity spectral filtering. A mode-locked laser output at a pulse repetition frequency $f$ (the period $T=1 / f$ ) reveals a discrete spectral comb with the frequency spacing $f$, as shown in Fig. 1. Using an appropriate filter, such as a Fabry-Pérot etalon, we can select periodic spectral lines with the frequency spacing $m f$, where $m$ is a positive integer. Then the resultant pulse speed increases $m$ times $(m f)$ when compare with the original speed, while the pulse period decreases to $T / m$ (see Fig. 1). In this way we can get a high-speed pulse train from a relatively slow pulse train by a simple spectral filtering.

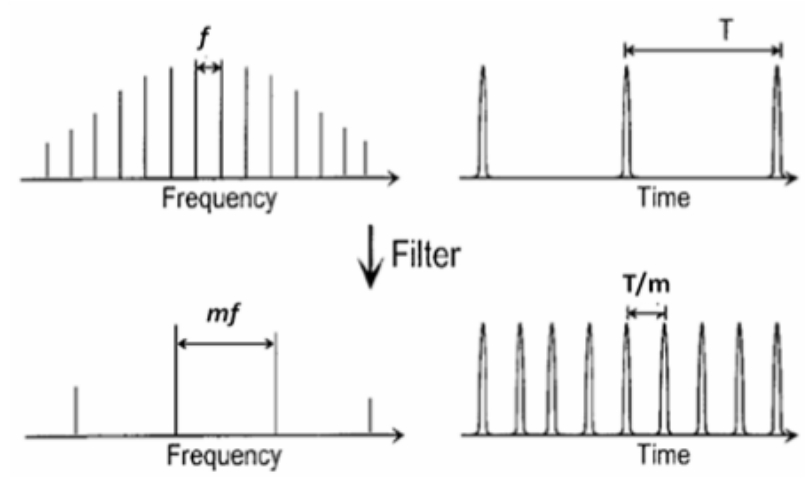

Fig. 1. Schematic diagram of high-speed pulse train generation by spectral filtering: spacing of the spectral comb increases by $m$ times when compare with the original spacing $f$, resulting in increasing pulse speed $(m f)$. 


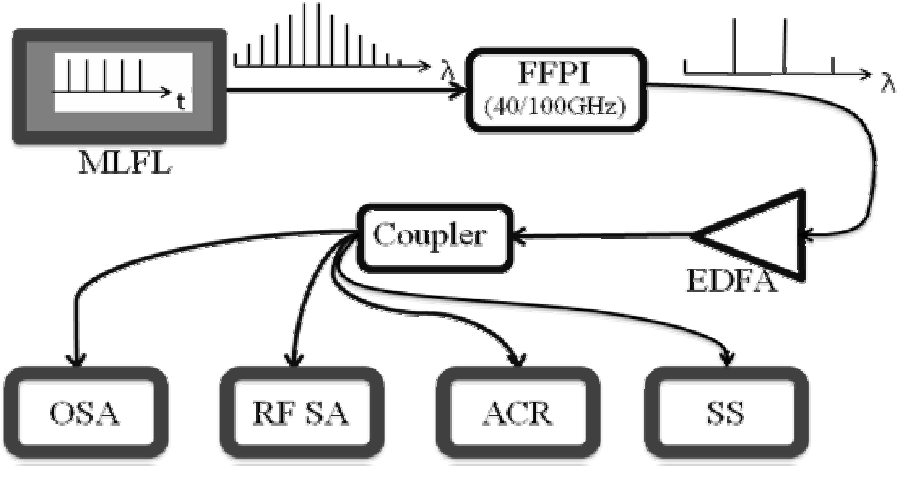

Fig. 2. Schematic diagram of our experimental setup.
Note that the envelope of the spectral comb does not change after the spectral filtering, ensuring the same pulse shape and width after multiplying $m$ times the pulse repetition rate.

Fig. 2 shows schematic experimental setup confirming our idea. A mode-locked fibre ring laser (MLFL) generates stable $10 \mathrm{GHz}$ pulse trains with 2 ps pulse width at $1550 \mathrm{~nm}$. For periodic spectral filtering, a MLFL output passes through a high-finesse ( 33) FFPI with the $40 \mathrm{GHz}$ or $100 \mathrm{GHz}$ FSR. After passing the FFPI, the output is amplified by an erbium-doped fibre amplifier and its characteristics are measured by an optical spectrum analyser, a radio frequency (RF) spectrum analyser coupled with a fast photodetector, an autocorrelator, and a fast sampling oscilloscope. The MLFL pulse frequency is tuned deliberately to match its harmonics with the FSR of the FFPI.

\section{Experimental results}

As discussed above, a $10 \mathrm{GHz}$ MLFL output is characterised by evenly spaced discrete spectral lines (an optical-frequency comb), with the frequency spacing equal to the pulse
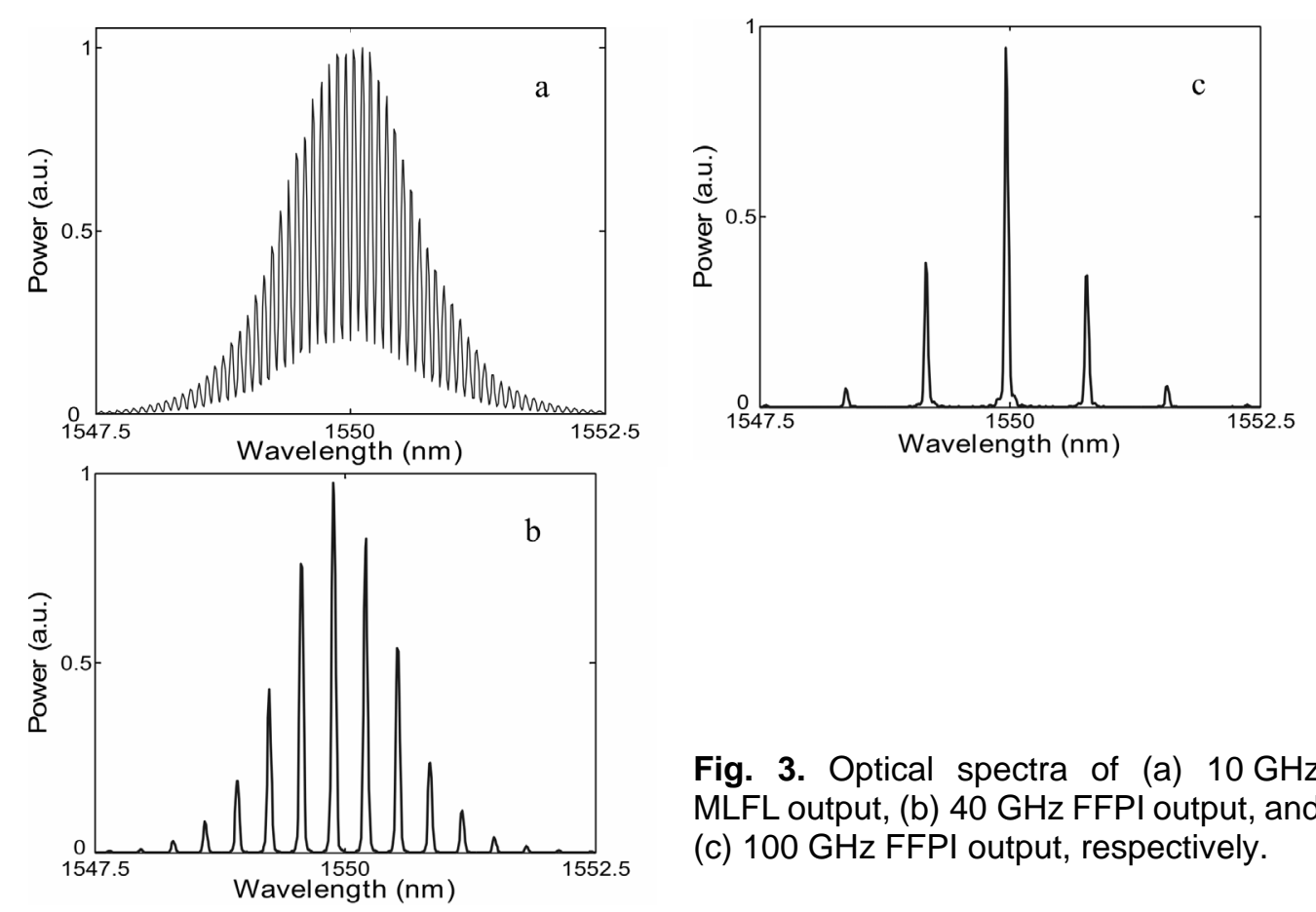

Fig. 3. Optical spectra of (a) $10 \mathrm{GHz}$ MLFL output, (b) $40 \mathrm{GHz}$ FFPI output, and (c) $100 \mathrm{GHz}$ FFPI output, respectively. 
repetition rate at $10 \mathrm{GHz}$. After passing the high-finesse FFPI with 40 or $100 \mathrm{GHz}$ FSR, we can filter out any unwanted spectral components from the $10 \mathrm{GHz}$ spectral comb, generating a new spectral comb with the $40 \mathrm{GHz}$ or $100 \mathrm{GHz}$ spacing, as demonstrated in Fig. 3b or Fig. 3c. As expected, the spectral envelopes of the 40 and $100 \mathrm{GHz}$ combs have their shapes very similar with that of $10 \mathrm{GHz}$, thus ensuring the same pulse shape/width after the spectral filtering, i.e. after the repetition rate multiplication.
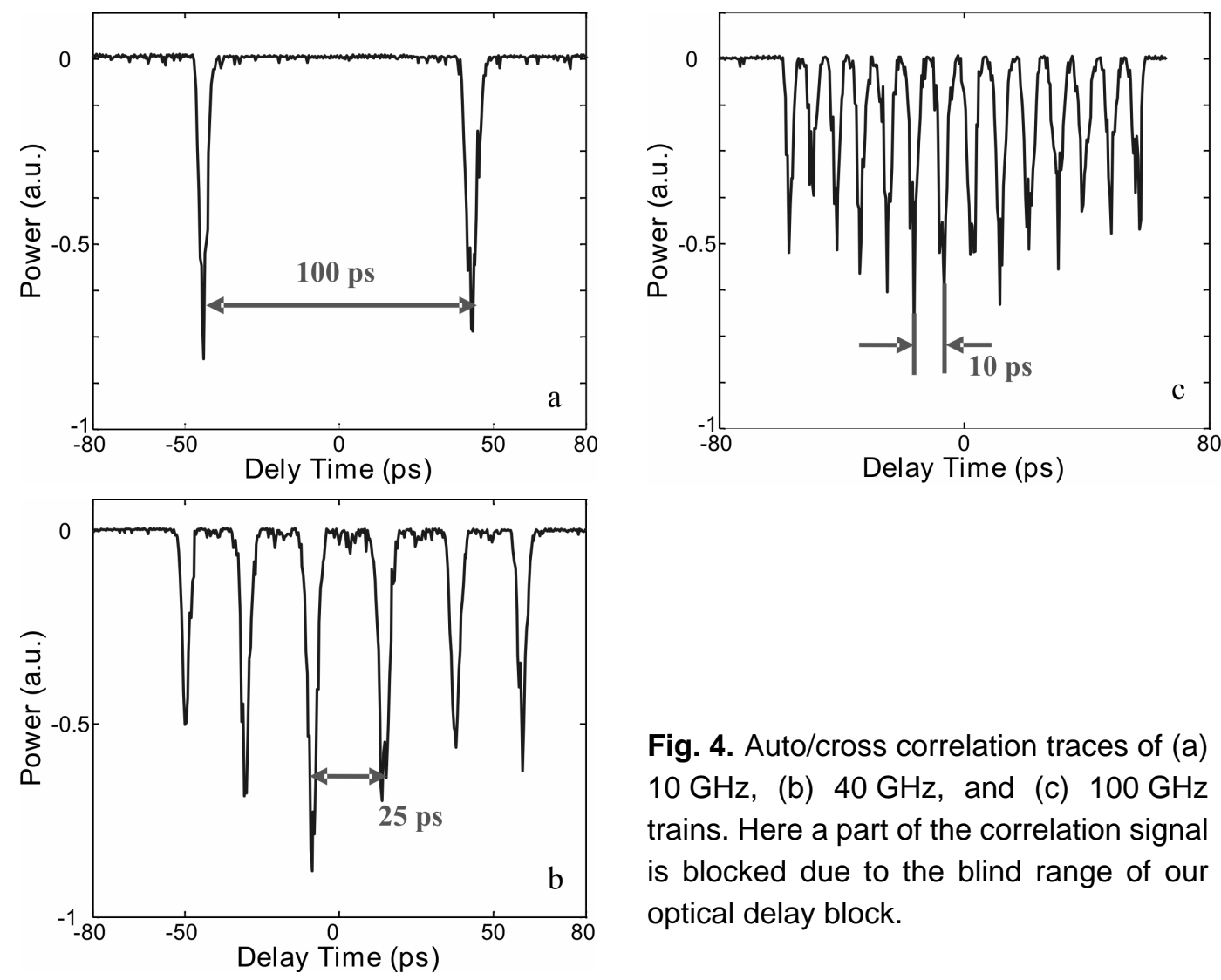

Fig. 4. Auto/cross correlation traces of (a) $10 \mathrm{GHz}$, (b) $40 \mathrm{GHz}$, and (c) $100 \mathrm{GHz}$ trains. Here a part of the correlation signal is blocked due to the blind range of our optical delay block.

To double confirm the fact of high-speed pulse train generation, we have measured the auto/cross correlation traces of the filtered outputs by delaying one of the correlation signals, as shown in Fig. 4. The results of those measurements substantiate that the pulse period is reduced from $100 \mathrm{ps}$ to $25 \mathrm{ps}$ or $10 \mathrm{ps}$. In addition, they also show the input and output pulse widths to be almost the same. Again we make sure that the $40 \mathrm{GHz}$ and $100 \mathrm{GHz}$ pulse trains are generated from the $10 \mathrm{GHz}$ MLFL by simple extra-cavity spectral filtering.

In order to check stability of the generated pulse train in details, we have detected the optical signal using a fast detector and examined its RF spectrum (see Fig. 5). For comparison, we have kept the same optical power of $1.5 \mathrm{dBm}$ at the RF spectrum analyser when measuring the RF spectra of the input and FPPI output pulse trains. Here the RF spectra are measured up to $25 \mathrm{GHz}$, the limit of our measurement facility. The $10 \mathrm{GHz}$ input pulses show clear $10 \mathrm{GHz}$ and its second harmonic components, as shown in Fig. 5a. 
Note that $10 \mathrm{GHz}$ and its harmonics (see Fig. 5b) are completely suppressed in the generated $40 \mathrm{GHz}$ pulses, showing clean enough pulses. However, a noticeable $10 \mathrm{GHz}$ component is observed in the $100 \mathrm{GHz}$ train, even though it is suppressed more than $20 \mathrm{~dB}$, as depicted in Fig. 5c. We think that the $10 \mathrm{GHz}$ component, indicating its periodic amplitude modulation at $10 \mathrm{GHz}$, is induced from the limited finesse of the $100 \mathrm{GHz}$ FFPI. This is understandable since the FFPI with broader FSR (for larger number of repetition rate multiplication) requires higher finesse to provide similar output quality. Similar envelope modulation, due to a finite finesse (or broad pass-band width) of a spectral filter, has been observed in the arrayed waveguide grating filter reported in [8].
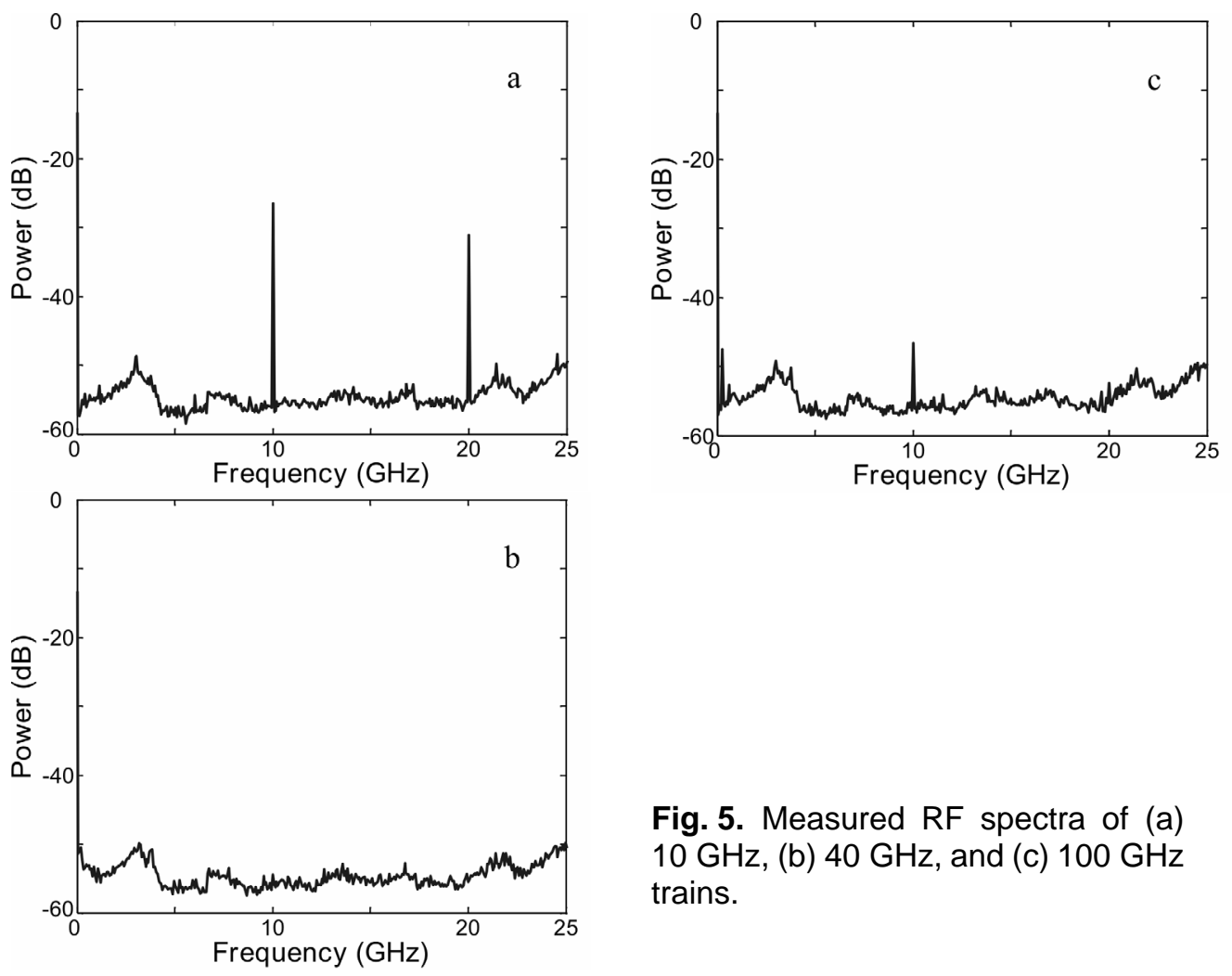

Fig. 5. Measured RF spectra of (a) $10 \mathrm{GHz}$, (b) $40 \mathrm{GHz}$, and (c) $100 \mathrm{GHz}$ trains.
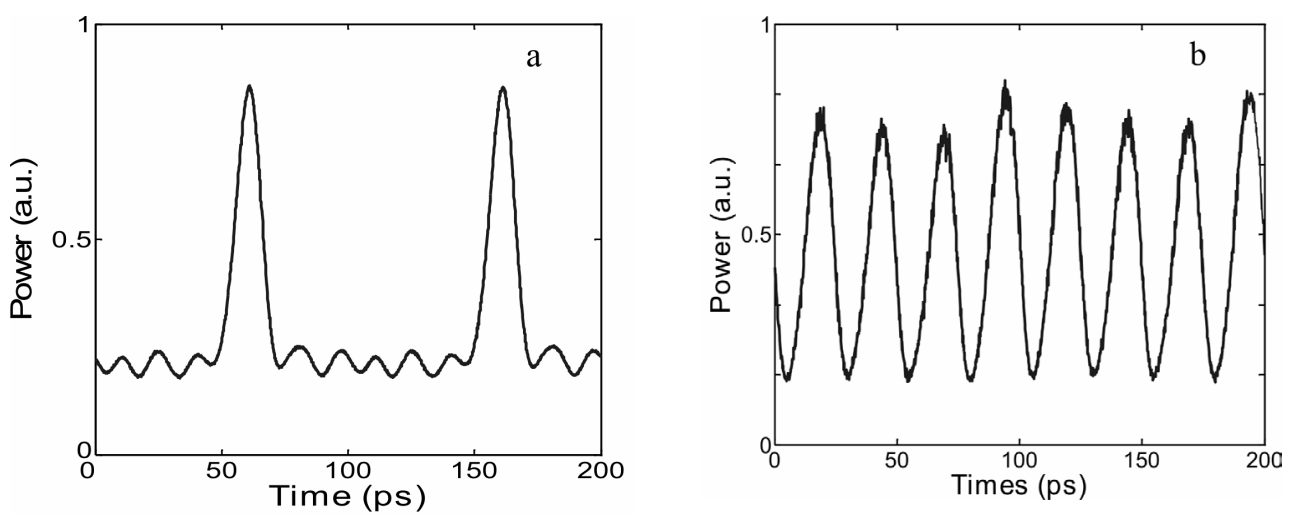

Fig. 6. Sample scope traces of (a) $10 \mathrm{GHz}$ and (b) $40 \mathrm{GHz}$ signals. 
We have also measured directly the time traces of the $10 \mathrm{GHz}$ input and the $40 \mathrm{GHz}$ FFPI output, using a $40 \mathrm{GHz}$ fast sampling scope (see Fig. 6). Again, reasonably stable $40 \mathrm{GHz}$ signal is observed. We believe that a small $10 \mathrm{GHz}$ amplitude fluctuation of the $40 \mathrm{GHz}$ train, which appears to be completely suppressed in the RF spectrum measurement (see Fig. 5b), is produced by some unknown $10 \mathrm{GHz}$ system operation noise and/or a limited FFPI finesse.

\section{Conclusion}

In this paper we have suggested a simple technique aimed at generation of high-speed pulse trains from relatively slow pulse sources, using spectral filtering with a high-finesse Fabry-Pérot interferometer for repetition rate multiplication. The FFPI selects a spectral comb with the frequency spacing corresponding to its FSR to generate a high-speed pulse train within its FSR. The optical spectra measured, autocorrelation traces, the RF spectra, and the sampling scope traces have revealed reasonably stable $40 \mathrm{GHz}$ and $100 \mathrm{GHz}$ pulse train generation from the $10 \mathrm{GHz}$ mode-locked laser by the spectral filtering technique suggested for repetition rate multiplication. The fundamental $10 \mathrm{GHz}$ component is suppressed completely in the $40 \mathrm{GHz}$ train and more than $20 \mathrm{~dB}$ in the $100 \mathrm{GHz}$ train. Other arbitrary-speed pulse trains at the harmonics of the input signal can be easily generated with the similar spectral filtering technique.

\section{Acknowledgment}

This work was supported by the Korea Research Foundation (\#2009-0072630).

\section{References}

1. Ahmed Z and Onodera N, 1996. High-repetition rate optical pulse generation by frequency multiplication in actively mode-locked fiber ring lasers. Electron. Lett. 32: 455-457.

2. Yoshida E and Nakazawa M, 1996. 80 200 GHz erbium doped fibre laser using a rational harmonic mode-locking technique. Electron. Lett. 32: 1370-1372.

3. Gupa K K and Onodera N, 2002. Pulse repetition frequency multiplication via intracavity optical filtering in AM mode-locked fiber ring lasers, IEEE Photon. Technol. Lett. 14: 284-286.

4. Abedin K S, Onodera N and Hyodo M, 1998. Repetition-rate multiplication in actively mode-locked fiber lasers by higher-order FM mode locking using a high-finesse Fabry-Perot filter. Appl. Phys. Lett. 73: 1311-1313.

5. Arahirea S, Kutsuzawa S, Matsui Y, Kunimatsu D and Ogawa Y, 1998. Repetition-frequency multiplication of mode-locked pulses using fiber dispersion. Lightwave. Technol. 16: 405-410.

6. Azana J, Kockaert P, Slavik R, Chen L R and Larochelle S, 2003. Generation of a 100 $\mathrm{GHz}$ optical pulse train by pulse repetition-rate multiplication using superimposed fiber Bragg gratings. IEEE Photon. Technol. Lett. 15: 413-415. 
7. McKinney J, Seo D S, Leaird D E and Weiner A M, 2003. Photonically Assisted Generation of Arbitrary Millimeter-wave and Microwave Electromagnetic Waveforms via Direct Space-to-Time Optical Pulse Shaping. Lightwave. Technol. 21: 3020-3022.

8. Seo D S, Leaird D E, Weiner A M, Kamei S, Ishii M, Sugita A and Okamoto K, 2003. Continuous $500 \mathrm{GHz}$ pulse train generation by repetition-rate multiplication using array, Electron. Lett. 39: 1138-1140.

9. Yiannopoulos K, Vyrsokinos K, Kehayas E, Pleros N, Vlachos K and Avramopoulos H, 2003. Rate multiplication by double-passing Fabry-pérot filtering. IEEE Photon. Technol. Lett. 15: 1294-1296.

Zhang Jia, Hong Sang-Jin and Seo Dong-Sun, 2010. High-speed pulse train generation by spectral filtering of a mode-locked laser output. Ukr.J.Phys.Opt. 11: 61-67.

Анотація. Запропоновано простий метод генерації високошвидкісних серій імпульсів від відносно низькошвидкісного імпульсного джерела шляхом спектрального фільтрування 3 використанням високодобротного волоконного інтерферометра Фабрі-Перо. Отримано стабільні серії імпульсів 40 ГГи і 100 ГГи з довжиною хвилі 1550 нм від 10 ГГи-волоконного кільчевого лазера з синхронізацією мод. 